\title{
EVALUATION OF EULER-ZAGIER SUMS
}

\section{KHRISTO BOYADZHIEV}

(Received 2 May 2000)

ABSTRACT. We present a simple method for evaluation of multiple Euler sums in terms of single and double zeta values.

2000 Mathematics Subject Classification. 11M99, $40 \mathrm{B05}$.

1. Introduction. We give a short evaluation of the triple sums

$$
w(p, q, r)=\sum_{n, m=1}^{\infty} \frac{1}{n^{p} m^{q}(n+m)^{r}}
$$

in terms of single zeta values $\zeta(p)$

$$
\zeta(p)=\sum_{n=1}^{\infty} n^{-p}
$$

and double zeta values (Euler sums) $S(p, q)$

$$
S(p, q)=\sum_{n=1}^{\infty} H_{n}^{(p)} n^{-q}, \quad H_{n}^{(p)}=1^{-p}+2^{-p}+\cdots+n^{-p},
$$

where $p \geq 1, q>1$.

Multiple Euler sums have been discussed and evaluated in a number of papers of which we want to point out [1, 2, 3, 4, 5, 6, 7, 10]. Also [8, Sections 18 and 19]. We refer to these publications for general comments and details.

\section{Euler sums}

LEMMA 2.1. For any integer $p>1$ and any $x>0$,

$$
\sigma(p ; x) \equiv \sum_{n=1}^{\infty} \frac{1}{n^{p}(n+x)}=\sum_{k=1}^{p-1} \frac{(-1)^{k-1} \zeta(p-k+1)}{x^{k}}+\frac{(-1)^{p-1}}{x^{p}}(\psi(x+1)+\gamma),
$$

where $\psi=\Gamma^{\prime} / \Gamma$ is the psi function and $\gamma$ is Euler's constant.

Proof. We have

$$
\begin{aligned}
\sigma(p ; x) & =\sum_{n=1}^{\infty} \frac{x+n-n}{n^{p} x(n+x)}=\sum_{n=1}^{\infty} \frac{1}{n^{p} x}-\sum_{n=1}^{\infty} \frac{1}{n^{p-1} x(n+x)} \\
& =\frac{1}{x}(\zeta(p)-\sigma(p-1 ; x)) .
\end{aligned}
$$


Equation (2.1) follows by repeating the procedure $p-1$ times in view of the fact that (see [9, page 665])

$$
\sigma(1 ; x)=\sum_{n=1}^{\infty} \frac{1}{n(n+x)}=\frac{1}{x}(\psi(x+1)+\gamma) .
$$

Now we differentiate (2.1) $r-1$ times, where $r>1$. With $D=d / d x$ we have

$$
\begin{gathered}
D^{r-1} \frac{1}{n+x}=\frac{(-1)^{r-1}(r-1) !}{(n+x)^{r}}, \\
D^{r-1} \frac{1}{x^{k}}=(-1)^{r-1} \frac{(r+k-2) !}{(k-1) !} \frac{1}{x^{r+k-1}}=(-1)^{r-1}(r-1) !\left(\begin{array}{c}
r+k-2 \\
r-1
\end{array}\right) \frac{1}{x^{r+k-1}}, \\
D^{r-1}\left(x^{-p}(\psi(x+1)+\gamma)\right)=\sum_{k=0}^{r-1}\left(\begin{array}{c}
r-1 \\
k
\end{array}\right)\left(D^{r-1-k} x^{-p}\right)\left(D^{k}(\psi(x+1)+\gamma)\right) .
\end{gathered}
$$

Therefore,

$$
\begin{aligned}
D^{r-1} \sigma(p ; x)= & (-1)^{r-1}(r-1) ! \sum_{n=1}^{\infty} \frac{1}{n^{p}(n+x)^{r}} \\
= & (-1)^{r-1}(r-1) ! \sum_{k=1}^{p-1}(-1)^{k-1}\left(\begin{array}{c}
r+k-2 \\
r-1
\end{array}\right) \frac{\zeta(p-k+1)}{x^{k+r-1}} \\
& +\frac{(-1)^{p-1}(-1)^{r-1}(r-1) !}{(p-1) !} \sum_{k=0}^{r-1} \frac{(-1)^{k}(r+p-k-2) !}{k !(r-k-1) !} \frac{(\psi(x+1)+\gamma)^{(k)}}{x^{r+p-k-1}} .
\end{aligned}
$$

We summarize this result in the following lemma.

LEMMA 2.2. For any integers $p>1, r \geq 1$ and any $x>0$,

$$
\begin{aligned}
\sum_{n=1}^{\infty} \frac{1}{n^{p}(n+x)^{r}}= & \sum_{k=1}^{p-1}(-1)^{k-1}\left(\begin{array}{c}
r+k-2 \\
r-1
\end{array}\right) \frac{\zeta(p-k+1)}{x^{k+r-1}} \\
& +\frac{(-1)^{p-1}}{(p-1) !} \sum_{k=0}^{r-1} \frac{(-1)^{k}(r+p-k-2) !}{k !(r-k-1) !} \frac{(\psi(x+1)+\gamma)^{(k)}}{x^{r+p-k-1}} .
\end{aligned}
$$

Next, we replace here $x$ by $m x$ and multiply both sides by $m^{-q}, q \geq 1$. This gives

$$
\begin{aligned}
\sum_{n=1}^{\infty} \frac{1}{n^{p} m^{q}(n+m x)^{r}}= & \sum_{k=0}^{p-2} \frac{(-1)^{k}}{x^{k+r}}\left(\begin{array}{c}
r+k-1 \\
r-1
\end{array}\right) \frac{\zeta(p-k)}{m^{k+q+r}} \\
& +\frac{(-1)^{p-1}}{(p-1) !} \sum_{k=0}^{r-1} \frac{(-1)^{k}(r+p-k-2) !}{k !(r-k-1) !} \frac{1}{x^{r+p-k-1}} \frac{(\psi(m x+1)+\gamma)^{(k)}}{m^{r+p+q-k-1}} .
\end{aligned}
$$

Summing for $m=1,2, \ldots$ we obtain our main representation. 
THEOREM 2.3. For all integers $p>1, r \geq 1$ and all $q \geq 0, q+r>1, x>0$,

$$
\begin{aligned}
\sigma(p, q, r ; x) \equiv & \sum_{m=1}^{\infty} \sum_{n=1}^{\infty} \frac{1}{n^{p} m^{q}(n+m x)^{r}} \\
= & \sum_{k=0}^{p-2} \frac{(-1)^{k}}{x^{k+r}}\left(\begin{array}{c}
r+k-1 \\
r-1
\end{array}\right) \zeta(p-k) \zeta(k+q+r) \\
& +\frac{(-1)^{p-1}}{(p-1) !} \sum_{k=0}^{r-1} \frac{(-1)^{k}(r+p-k-2) !}{k !(r-k-1) !} \frac{1}{x^{r+p-k-1}} \sum_{m=1}^{\infty} \frac{(\psi(m x+1)+\gamma)^{(k)}}{m^{r+p+q-k-1}} .
\end{aligned}
$$

The case $p=1$ can be derived directly from (2.3), namely,

$$
\begin{aligned}
\sigma(1, q, r ; x) & =\sum_{m=1}^{\infty} \sum_{n=1}^{\infty} \frac{1}{n m^{q}(n+m x)^{r}} \\
& =\sum_{k=0}^{r-1} \frac{(-1)^{k}}{k !} \frac{1}{x^{r-k}} \sum_{m=1}^{\infty} \frac{(\psi(m x+1)+\gamma)^{(k)}}{m^{r+q-k}} .
\end{aligned}
$$

We remind the reader that the expression $(\psi(m x+1)+\gamma)^{(k)}$ stands for the $k$ th derivative of the function $\psi(x+1)+\gamma$ evaluated at $m x$.

By setting $x=1$ we get the desired representation of $w(p, q, r)$. Making use of

$$
\begin{aligned}
& \psi(m+1)+\gamma=H_{m}^{(1)}=1+2^{-1}+\cdots+m^{-1}, \\
& \psi^{(k)}(m+1)=(-1)^{k} k !\left[H_{m}^{(k+1)}-\zeta(k+1)\right],
\end{aligned}
$$

(see [9, page 775$]$ ), and with the agreement to $\operatorname{read} \zeta(1)=0$, one obtains the following corollary.

COROLlaRY 2.4. For all integers $p>1, r \geq 1$ and all $q \geq 0$ with $q+r>1$,

$$
\begin{aligned}
w(p, q, r)= & \sum_{m=1}^{\infty} \sum_{n=1}^{\infty} \frac{1}{n^{p} m^{q}(n+m)^{r}} \\
= & \sum_{k=0}^{p-2}(-1)^{k}\left(\begin{array}{c}
r+k-1 \\
r-1
\end{array}\right) \zeta(p-k) \zeta(r+q+k) \\
& +\frac{(-1)^{p-1}}{(p-1) !} \sum_{k=0}^{r-1} \frac{(r+p-k-2) !}{(r-k-1) !}[S(k+1, r+p+q-k-1)-\zeta(k+1) \zeta(r+p+q-k-1)],
\end{aligned}
$$

in particular,

$$
\begin{aligned}
w(1, q, r) & =\sum_{m=1}^{\infty} \sum_{n=1}^{\infty} \frac{1}{n m^{q}(n+m)^{r}} \\
& =\sum_{k=0}^{r-1}[S(k+1, r+q-k)-\zeta(k+1) \zeta(r+q-k)] .
\end{aligned}
$$


When $q>0$ (or $q \geq 1, p=1$ ) we also have

$$
\begin{aligned}
w(p, q, 1) & =\sum_{m=1}^{\infty} \sum_{n=1}^{\infty} \frac{1}{n^{p} m^{q}(n+m)} \\
& =\sum_{k=1}^{p-1}(-1)^{k-1} \zeta(p-k+1) \zeta(q+k)+(-1)^{p-1} S(1, p+q) .
\end{aligned}
$$

3. Remarks. Our notation $S(p, q)$ corresponds to $S_{p, q}$ in [5]. The authors of [2] use the sums $\zeta(p, q)$, which equal $S(q, p)-\zeta(p+q)$.

The representation (2.11) has strong and weak points. One good feature is that $q$ need not be an integer. A weak point is that the right-hand side in (2.11) is not explicitly symmetrical in $p$ and $q$, while obviously $w(p, q, r)=w(q, p, r)$. Moreover, the right-hand side has too many terms. For instance, when $q=0$ (2.11) becomes

$$
\begin{aligned}
w(p, 0, r)= & \sum_{m=1}^{\infty} \sum_{n=1}^{\infty} \frac{1}{n^{p}(n+m)^{r}} \\
= & (-1)^{p-1} S(r, p)+\sum_{k=0}^{p-2}(-1)^{k}\left(\begin{array}{c}
r+k-1 \\
r-1
\end{array}\right) \zeta(p-k) \zeta(r+k) \\
& +\frac{(-1)^{p-1}}{(p-1) !} \sum_{k=0}^{r-2} \frac{(r+p-k-2) !}{(r-k-1) !}[S(k+1, r+p-k-1)-\zeta(k+1) \zeta(r+p-k-1)]
\end{aligned}
$$

(here the term $(-1)^{p-1} S(r, p)$ is written separately on purpose).

At the same time

$$
w(p, 0, r)=\sum_{n=1}^{\infty} \frac{1}{n^{p}} \sum_{m=1}^{\infty} \frac{1}{(n+m)^{r}}=\sum_{n=1}^{\infty} \frac{1}{n^{p}}\left(\zeta(r)-H_{n}^{(r)}\right)=\zeta(p) \zeta(r)-S(r, p)
$$

which is much shorter. However, we can benefit from this situation if we compare the two representations of $w(p, 0, r)$ and derive relations for the single and double Euler sums. For instance, when $p$ is odd, we can solve for $S(r, p)$ to get

$$
\begin{aligned}
2 S(r, p)= & \sum_{k=1}^{p-2}(-1)^{k+1}\left(\begin{array}{c}
r+k-1 \\
r-1
\end{array}\right) \zeta(p-k) \zeta(r+k) \\
& +\frac{(-1)^{p-1}}{(p-1) !} \sum_{k=0}^{r-2} \frac{(r+p-k-2) !}{(r-k-1) !}[S(k+1, r+p-k-1)-\zeta(k+1) \zeta(r+p-k-1)],
\end{aligned}
$$

that is, $S(r, p)$ can be expressed in terms of single zeta values and $S(k, l)$, with $k<r$, $k+l=r+p$.

4. Other sums. It is interesting to consider also the sum

$$
u(p, q, r)=\sum_{n=1}^{\infty} \sum_{m=1}^{\infty} \frac{1}{n^{p} m^{q}\left(n^{r}+m^{r}\right)}
$$


and compare it to $w(p, q, r)$. Here one can write

$$
u(p, q, r) \sum_{n=1}^{\infty} \sum_{m=1}^{\infty} \frac{n^{r}+m^{r}-n^{r}}{n^{p+r} m^{q}\left(n^{r}+m^{r}\right)}=\zeta(p+r) \zeta(q)-u(p+r, q-r, r) .
$$

Let $q>p$. We observe that if $(q-p) / r$ is odd, repeating this step $(q-p) / r$ times, we get

$$
u(p, q, r)=\sum_{j=1}^{(q-p) / r}(-1)^{j-1} \zeta(p+j r) \zeta(q-(j-1) r)-u(q, p, r)
$$

from where, because of the symmetry $u(p, q, r)=u(q, p, r)$, we obtain the following proposition.

Proposition 4.1. For all $q>p \geq 1, r \geq 1$ with $(q-p) / r$ odd,

$$
u(p, q, r)=\frac{1}{2} \sum_{j=1}^{(q-p) / r}(-1)^{j-1} \zeta(p+j r) \zeta(q-(j-1) r) .
$$

Note that $p, q, r$ need not be integers. The only restrictions are those listed above. When $r=1$ we have

$$
\sum_{n=1}^{\infty} \sum_{m=1}^{\infty} \frac{1}{n^{p} m^{q}(n+m)}=\frac{1}{2} \sum_{j=1}^{q-p}(-1)^{j-1} \zeta(p+j) \zeta(q-j+1)
$$

which can be compared to (2.13). This gives the well-known expression of $S(1, p+q)$ in terms of zeta values. To make this more explicit we set $p=1$ and $q \geq 2$. Then from (2.13),

$$
w(1, q-1,1)=\sum_{m=1}^{\infty} \sum_{n=1}^{\infty} \frac{1}{n m^{q-1}(n+m)}=S(1, q) .
$$

This is the same as $u(1, q-1,1)$. When $q$ is odd we find from (4.5) (with $p=1$ and $q-1$ in the place of $q$ )

$$
S(1, q)=\frac{1}{2} \sum_{j=1}^{q-2}(-1)^{j-1} \zeta(j+1) \zeta(q-j)
$$

which is a variant of Euler's formula for the sum $S(1, q)$ (see [5, Theorem 2.2]).

\section{REFERENCES}

[1] J. M. Borwein, D. M. Bradley, and D. J. Broadhurst, Evaluations of k-fold Euler/Zagier sums: a compendium of results for arbitrary $k$, J. Combin 4 (1997), no. 2, 31-49. Zbl 884.40004.

[2] J. M. Borwein and R. Girgensohn, Evaluation of triple Euler sums, Electron. J. Combin. 3 (1996), no. 1, 1-27. MR 97d:11137. Zbl 884.40005.

[3] R. E. Crandall, Fast evaluation of multiple zeta sums, Math. Comp. 67 (1998), no. 223, 1163-1172. MR 98j:11066. Zbl 901.11036.

[4] R. E. Crandall and J. P. Buhler, On the evaluation of Euler sums, Experiment. Math. 3 (1994), no. 4, 275-285. MR 96e:11113. Zbl 833.11045.

[5] P. Flajolet and B. Salvy, Euler sums and contour integral representations, Experiment. Math. 7 (1998), no. 1, 15-35. MR 99c:11110. Zbl 920.11061. 
[6] A. Granville, A decomposition of Riemann's zeta-function, Analytic Number Theory (Kyoto, 1996) (Y. Motohashi, ed.), London Math. Soc. Lecture Note Ser., vol. 247, Cambridge Univ. Press, Cambridge, 1997, pp. 95-101. MR 2000c:11134. Zbl 907.11024.

[7] C. Markett, Triple sums and the Riemann zeta function, J. Number Theory 48 (1994), no. 2, 113-132. MR 95f:11067. Zbl 0810.11047.

[8] N. Nielsen, Die Gammafunktion, Chelsea, New York, 1965 (German). MR 32\#2622.

[9] A. P. Prudnikov, Y. A. Brychkov, and O. I. Marichev, Integrals and Series, Elementary functions, vol. 1, Gordon and Breach Science Publishers, New York, 1986. MR 88f:00013. Zbl 733.00004 .

[10] D. Zagier, Values of zeta functions and their applications, First European Congress of Mathematics, vol. II (Paris, 1992) (A. Joseph et al., ed.), Progr. Math., vol. 120, Birkhäuser, Basel, 1994, pp. 497-512. MR 96k:11110. Zbl 822.11001.

Khristo Boyadzhiev: Department of MATHEMATICS, OHIO NORTHERn UNIVERSity, ADA, OH 45810, USA

E-mail address: k-boyadzhi ev@onu.edu 


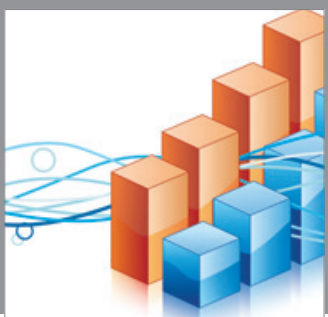

Advances in

Operations Research

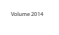

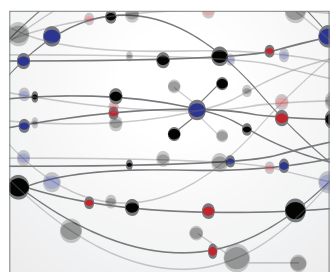

\section{The Scientific} World Journal
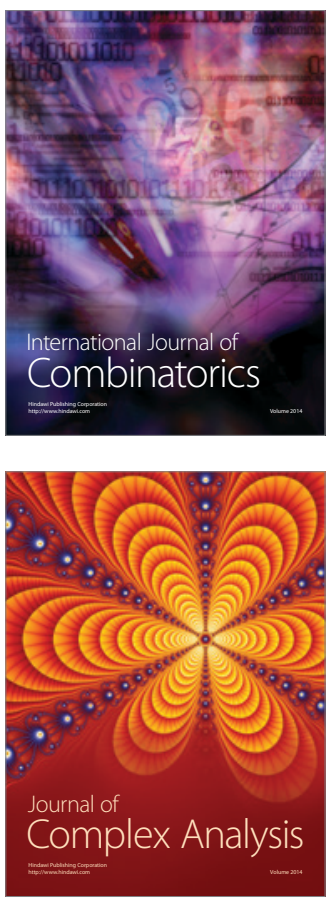

International Journal of

Mathematics and

Mathematical

Sciences
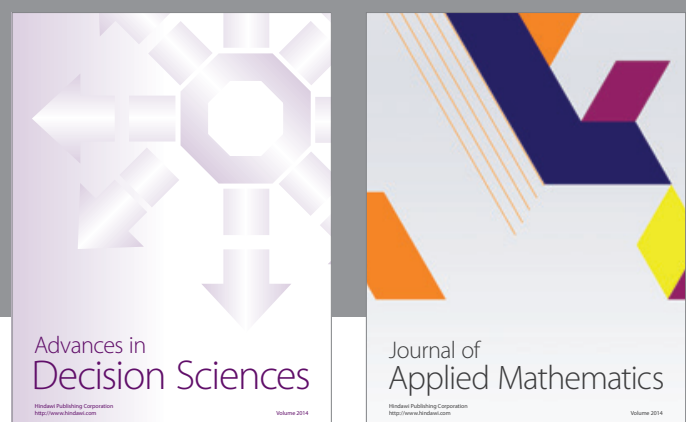

Journal of

Applied Mathematics
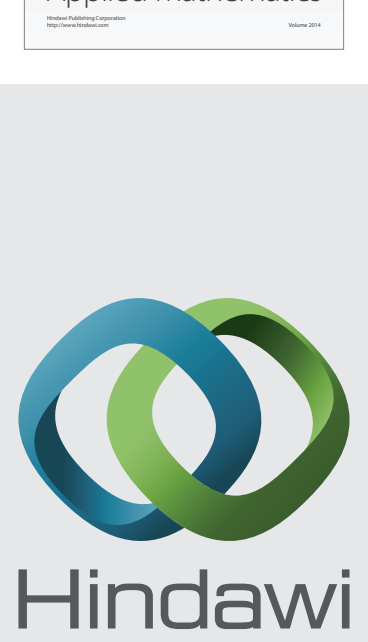

Submit your manuscripts at http://www.hindawi.com
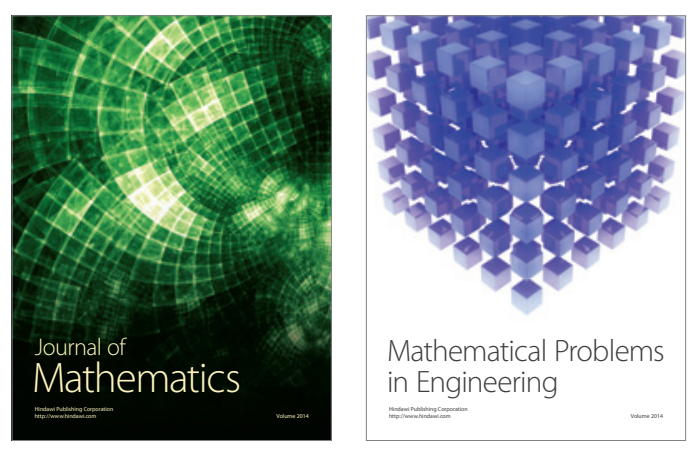

Mathematical Problems in Engineering
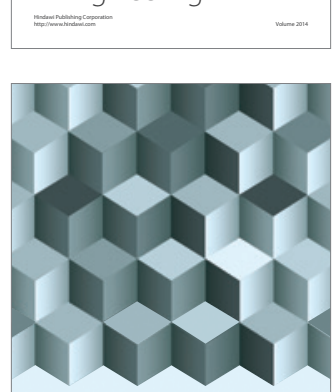

Journal of

Function Spaces
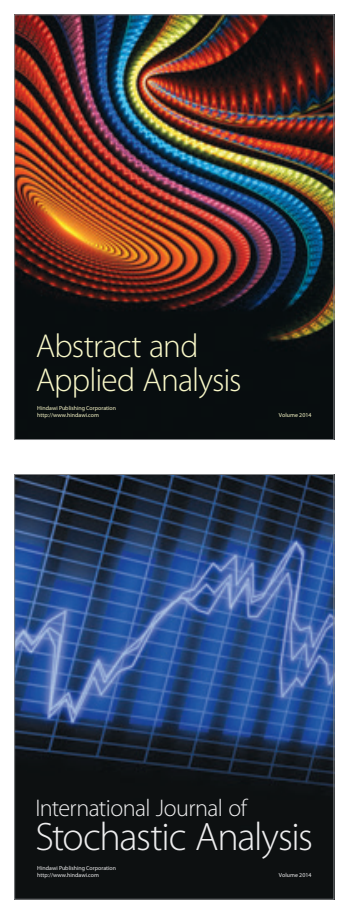

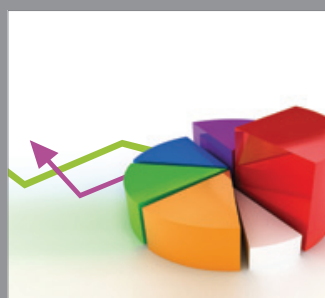

ournal of

Probability and Statistics

Promensencen
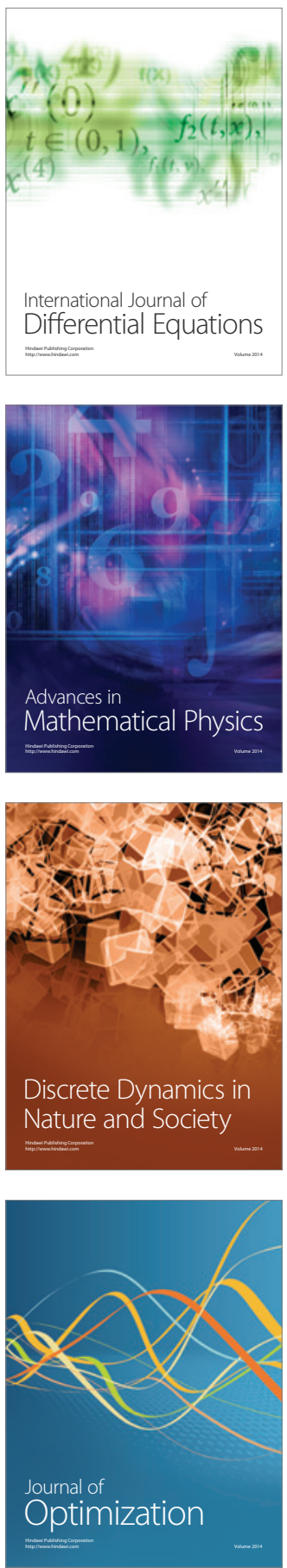